MATHEMATICS OF COMPUTATION

Volume 74, Number 252, Pages 2027-2033

S 0025-5718(05)01705-9

Article electronically published on March 24, 2005

\title{
INVOLUTIONS AND CHARACTERS OF UPPER TRIANGULAR MATRIX GROUPS
}

\author{
I. M. ISAACS AND DIKRAN B. KARAGUEUZIAN
}

\begin{abstract}
We study the realizability over $\mathbb{R}$ of representations of the group $U(n)$ of upper-triangular $n \times n$ matrices over $\mathbb{F}_{2}$. We prove that all the representations of $U(n)$ are realizable over $\mathbb{R}$ if $n \leq 12$, but that if $n \geq 13, U(n)$ has representations not realizable over $\mathbb{R}$. This theorem is a variation on a result that can be obtained by combining work of J. Arregi and A. Vera-López and of the authors, but the proof of the theorem in this paper is much more natural.
\end{abstract}

\section{INTRODUCTION}

Let $U(n)$ be the group of upper-triangular $n \times n$ matrices over the field of two elements. In this paper we prove

Theorem 1.1. If $n \leq 12$, every representation of $U(n)$ is realizable over $\mathbb{R}$.

We also discuss the complementary result:

Theorem 1.2. If $n \geq 13$, then $U(n)$ has an irreducible representation whose character is not real valued; this representation is in particular not realizable over $\mathbb{R}$.

These results lead naturally to the following.

Problem 1.3. Is every representation of $U(n)$, whose character is real valued, realizable over $\mathbb{R}$ ?

We remind the reader that a representation realizable over $\mathbb{R}$ has a real-valued character, but the converse is not true. (Consider the quaternion group of order 8.) In other words, a positive answer to the question above would be specific to $U(n)$. A conjecture on a special case of this problem will be presented in Section 4 .

One might also ask how the numbers 12 and 13 arise in Theorems 1.1 and 1.2 . Perhaps the main virtue of this paper is that our results obtain these numbers in a much simpler way than was previously possible.

The qualitative difference between the representation theory of $U(n)$ for $n \leq 12$ and the theory for $n \geq 13$ has been known for some time. A result highlighting this difference can be obtained by combining work of J. Arregi and A. Vera-López [8, 9] with work of the present authors [5, 6].

Received by the editor August 6, 2002 and, in revised form, February 24, 2004.

2000 Mathematics Subject Classification. Primary 20C15; Secondary 20D15.

Key words and phrases. Character theory, finite groups, p-groups.

The research of the second author was partially supported by an N.S.F. Postdoctoral Fellowship, the MPIM-Bonn, and the CRM-Barcelona. 
Theorem 1.4. If $n \leq 12$, every character of $U(n)$ is real valued, while if $n \geq 13$, $U(n)$ has characters that are not real valued.

The proof, which will be described later in this section, is extremely awkward. By contrast, we will give a simple proof of the main result of this paper:

Theorem 1.5. If $n \leq 12$, every representation of $U(n)$ is realizable over $\mathbb{R}$, while if $n \geq 13, U(n)$ has representations not realizable over $\mathbb{R}$.

To prove this result, we develop a recursive algorithm to count the involutions in $U(n)$ and prove our theorem by comparing the result of this count with the sum of the irreducible character degrees of $U(n)$, computed using an algorithm of M. Slattery [7.

These two results (Theorems 1.4 and 1.5) are of course related, since a representation realizable over $\mathbb{R}$ has a real-valued character. Thus, the $n \leq 12$ case of Theorem 1.4 is weaker than the $n \leq 12$ case of Theorem 1.5. but for $n \geq 13$, the reverse is true.

Since the two results are so similar, it is interesting to see how very different their proofs are, and so we give a brief description of the argument that establishes Theorem 1.4. Recall that an element $g$ of an arbitrary finite group $G$ is conjugate to its inverse if and only if $\chi(g)$ is real for all irreducible characters $\chi$ of $G$. (This is immediate from the second orthogonality relation [4, Theorem 2.18].) To prove Theorem 1.4, therefore, it suffices to find an element of $U(13)$ that is not conjugate to its inverse and to show that no such element exists in $U(12)$. The required element of $U(13)$ was found [5] using a random search and the nonexistence of such elements in $U(12)$ was established 9 by exhaustion. The computation in [9] was made possible by the fact that it suffices to check just one element in each conjugacy class. The $2^{66}$ elements of $U(12)$ are divided into only $34,064,872$ classes, and according to [8], each class contains a matrix in an appropriate canonical form.

The argument sketched in the previous paragraph hardly seems a natural approach to the problem; the motivation comes from a long study of the character theory of these groups. The method of proof of Theorem 1.5. by contrast, is straightforward. A standard result of character theory (Corollary 3.2) tells us that the representations of a group are all realizable over $\mathbb{R}$ if and only if one plus the number of involutions is equal to the sum of the character degrees. We develop in this paper a function to count the involutions in a class of groups containing $U(n)$. An algorithm for computing the character degrees was developed by Slattery [7. Thus, to check whether Theorem 1.5 is true for a given $n$, we just need to check whether two numbers are equal.

Furthermore, it is a consequence of the "canonical form" results and computations of Arregi and Vera-Lopez that $U(13)$ has exactly one complex-conjugate pair of characters that are not real valued. Combining this result with the methods of the present paper, we are able to prove:

Proposition 1.6. If $\theta$ is a character of $U(13)$ that is not real valued, $\theta(1) \leq 2^{16}$.

As remarked earlier in this Introduction, Theorem 1.5 and Proposition [1.6 are proved using (among other tools) an algorithm for counting involutions; the development of this algorithm occupies Section 2 of this paper. In Section 3 we discuss the application of the algorithm to character theory and prove Theorem 1.5 and Proposition [1.6. In the last section there are some remarks about the motivation behind the proof of these results. 
The authors are pleased to acknowledge a number of helpful conversations with Andrea Previtali.

\section{A ReCURSive Algorithm FOR COUNTing INVOLUtions}

In this section we develop an algorithm for counting the number of involutions (elements of order 2) in the group $U(n)$ of unipotent upper triangular matrices in $G L_{n}(q)$, where $q$ is a power of 2 .

If $G$ is a finite group, we make the following

Definition 2.1. We write $t(G)$ for the cardinality of the set $\left\{x \in G \mid x^{2}=1\right\}$.

Note that $t(G)$ is one more than the number of involutions of $G$; we abbreviate $t(U(n))$ to $t(n)$.

If $A$ is a matrix in $U(n)$, we can write $A=1+X$, where $X$ is a strictly upper triangular matrix. We want to count the number $t(n)$ of elements $A \in U(n)$ such that $A^{2}=1$. Since $A=1+X$ and we are working in characteristic 2 , we see that $A^{2}=1+X^{2}$, and so our task is to count the strictly upper triangular matrices $X$ such that $X^{2}=0$. We will count these matrices recursively, partitioning them by both size and rank.

Definition 2.2. We write $f_{r}(n)$ for the number of strictly upper triangular $n \times n$ matrices $X$ with entries in the field of order $q$ and $\operatorname{rank} X=r$ such that $X^{2}=0$. Note that $f_{r}(n)$ is defined only for $n \geq 1$ and $r \geq 0$.

Because our primary interest is in the representation-theoretic results stated in the introduction, our real goal is to compute $t(n)$. We note that we can count the matrices by rank, i.e., $t(n)=\sum_{r=0}^{n} f_{r}(n)$. In fact, we can refine this equation slightly to

$$
t(n)=\sum_{r=0}^{\left\lfloor\frac{n}{2}\right\rfloor} f_{r}(n)
$$

using the following.

Lemma 2.4. $f_{r}(n)=0$ if $r>\frac{n}{2}$.

Proof. We view an $n \times n$ matrix $X$ as a right operator and write $R(X)$ for the row space (or image) of $X$ and $K(X)$ for the kernel of $X$. We have $\operatorname{dim} R(X)+$ $\operatorname{dim} K(X)=n$, and $\operatorname{dim} R(X)=\operatorname{rank} X$ by definition of rank. A matrix $X$ for which $X^{2}=0$ satisfies $R(X) \subseteq K(X)$, so for such a matrix $\operatorname{dim} K(X) \geq \operatorname{dim} R(X)$. Combining these two facts shows that if $X^{2}=0$, then $\operatorname{rank} X \leq \frac{n}{2}$.

The main result of this section is that $f_{r}(n)$ satisfies the following recurrence relation. (Recall that $q$ is the order of the field in which the entries of the matrices lie.)

Theorem 2.5. If $n>1$ and $r>0$, then

$$
f_{r}(n)=q^{r} \cdot f_{r}(n-1)+\left(q^{n-r}-q^{r-1}\right) \cdot f_{r-1}(n-1) .
$$

Remark 2.6. Note that if the coefficient $\left(q^{n-r}-q^{r-1}\right)$ is negative, then $n-r<r-1$, and thus $2 r>n+1$ and we have $2(r-1)>(n-1)$. In this case, however, Lemma 2.4 shows that $f_{r-1}(n-1)=0$, and thus negative coefficients never really occur in the equation of Theorem 2.5 . 
We now turn to the proof of the recurrence relation.

Proof of Theorem 2.5. Since $n>1$, we can think of the matrix $X$ as being decomposed like

$$
X=\left[\begin{array}{cc}
0 & v \\
0 & Y
\end{array}\right],
$$

where $v$ is a row vector of length $n-1$ and $Y$ is a strictly upper triangular $(n-1) \times(n-1)$ matrix. Note that the lower left entry in the matrix above is the zero column vector of length $n-1$.

Assuming that $X$ is decomposed as above, we see that

$$
X^{2}=\left[\begin{array}{cc}
0 & v Y \\
0 & Y^{2}
\end{array}\right]
$$

and so $X^{2}=0$ if and only if $Y^{2}=0$ and $v Y=0$. Assuming (recursively) that we can find the matrices $Y$ such that $Y^{2}=0$, we see that we need to count the row vectors $v$ such that $v Y=0$. These, of course, are exactly the vectors of the nullspace $K(Y)$ of $Y$, viewed as a right operator. Since we are concerned only with matrices $Y$ such that $Y^{2}=0$, just as in the proof of Lemma 2.4 we see that each row of $Y$ lies in $K(Y)$, and thus we can write $R(Y) \subseteq K(Y)$, where $R(Y)$ is the row space of $Y$.

Again assume that $n>1$ and observe that the matrices $X$ that we want to count come in two flavors: those where $v \in R(Y)$ and those where $v \notin R(Y)$. For matrices of the first type, the first row of $X$ is a member of the space spanned by the other rows of $X$, and thus the ranks of $X$ and $Y$ are equal. For matrices of the second type, the first row of $X$ does not lie in the span of the remaining rows and thus $\operatorname{rank} X=\operatorname{rank} Y+1$.

Now $f_{r}(n)$ is the sum of the numbers of square zero rank $r$ strictly upper triangular $n \times n$ matrices of each of the two types. The number of such matrices of the first type is the number of square zero matrices $Y$ with rank $r$ times the number of row vectors $v$ in the row space of $Y$. This component of $f_{r}(n)$ is equal to $q^{r} \cdot f_{r}(n-1)$. The remaining matrices $X$ come from matrices $Y$ of rank $r-1$ with vectors $v$ in $K(Y)$ but not in $R(Y)$. The number of such vectors $v$ is $q^{(n-1)-(r-1)}-q^{r-1}$. This yields the recurrence relation.

In order to get our computations started, we need some "initial" values of $f_{r}(n)$.

Lemma 2.7. For all $r \geq 1, f_{r}(1)=0$. For all $n \geq 1, f_{0}(n)=1$.

Proof. These equalities hold because no $1 \times 1$ matrix with square 0 has rank 1 and only the zero matrix has rank 0 .

Theorem 2.5 and Lemma 2.7 can be used to compute all of the values of $f_{r}(n)$ for $n \geq 1$. Table 1 lists some of these values in the case where $q=2$. The entries in each row of the table are the nonzero values of $f_{r}(n)$ for $r \geq 0$. Of course, the number $t(n)$ can be computed by taking the sum (equation 2.3) of the $n$th row in a table like this. 
TABLE 1.

\begin{tabular}{c|ccccccc}
$n$ & $f_{0}(n)$ & $f_{1}(n)$ & $f_{2}(n)$ & $f_{3}(n)$ & $f_{4}(n)$ & $f_{5}(n)$ & \\
\hline 1 & 1 & & & & & & \\
2 & 1 & 1 & & & & & \\
3 & 1 & 5 & & & & & \\
4 & 1 & 17 & 10 & & & & \\
5 & 1 & 49 & 142 & & & & \\
6 & 1 & 129 & 1254 & 568 & & & \\
7 & 1 & 321 & 8886 & 19592 & & & \\
8 & 1 & 769 & 55446 & 405544 & 156736 & & \\
9 & 1 & 1793 & 318678 & 6571112 & 12240832 & & \\
10 & 1 & 4097 & 1730134 & 92084968 & 563835584 & 195853312 & \\
11 & 1 & 9217 & 9010006 & 1172673512 & 20071565504 & 33331414016 & \\
12 & 1 & 20481 & 45459798 & 13958471144 & 611968079040 & 3314620584960 & 1066605248512 \\
13 & 1 & 45057 & 223743318 & 158036763112 & 16826558721216 & 252940197688320 & 386466312060928
\end{tabular}

\section{Applichtions to Character theory}

In this section we use the results developed in Section 2 to prove the theorems stated in the Introduction. This means that throughout this section, we take the field $k$ to be $\mathbb{F}_{2}$.

We begin by quoting a result from character theory. Recall from [4, Corollary 4.6] the following.

Proposition 3.1. For any finite group $G$,

$$
t(G)=\sum_{\chi \in \operatorname{Irr}(G)} \nu_{2}(\chi) \chi(1)
$$

where $\nu_{2}(\chi)=0$ if $\chi$ is not real valued, $\nu_{2}(\chi)=1$ if $\chi$ is realizable over $\mathbb{R}$, and $\nu_{2}(\chi)=-1$ if $\chi$ is real valued but not realizable over $\mathbb{R}$.

As an immediate consequence we have:

Corollary 3.2. All the representations of $G$ are realizable over $\mathbb{R}$ if and only if $t(G)=\sum_{\chi \in \operatorname{Irr}(G)} \chi(1)$.

Theorem 1.5 will follow from this corollary once we have computed the numbers on both sides of the equation (in 3.2).

From the table at the end of the previous section we can obtain $t(n)$; using Slattery's algorithm [7, which is implemented in MAGMA 11, we obtain Table 2 (for the case $k=\mathbb{F}_{2}$ ).

TABLE 2 .

\begin{tabular}{c|c|c}
$n$ & $t(n)$ & $\sum_{\chi \in \operatorname{Irr}(U(n))} \chi(1)$ \\
\hline 2 & 2 & 2 \\
3 & 6 & 6 \\
4 & 28 & 28 \\
5 & 192 & 192 \\
6 & 1952 & 1952 \\
7 & 28800 & 28800 \\
8 & 618496 & 618496 \\
9 & 19132416 & 19132416 \\
10 & 853508096 & 853508096 \\
11 & 54584672256 & 54584672256 \\
12 & 5007197863936 & 5007197863936 \\
13 & 656391329021952 & 656391329153024
\end{tabular}


This of course allows us to prove Theorem 1.5.

Theorem 1.5. If $n \leq 12$, every representation of $U(n)$ is realizable over $\mathbb{R}$, while if $n \geq 13, U(n)$ has representations not realizable over $\mathbb{R}$.

Proof. To prove the $n \leq 12$ part of the theorem, observe that if $n \leq 12$ the numbers in the second and third columns of the appropriate line in Table 2 are the same. Corollary 3.2 then gives the realizibility. For $n=13$, this follows from the $n=13$ line of Table 2 and Corollary [3.2. For $n>13$, note that there are surjective homomorphisms $U(n) \rightarrow U(n-1)$.

Arregi and Vera-López were able to show, using their results on canonical forms, that there is exactly one pair of conjugacy classes of elements of $U(13)$ that are not conjugate to their inverses [9]. It follows from a theorem of Brauer 4, Theorem 6.32, Exercise 6.13] that there is exactly one complex-conjugate pair of characters of $U(13)$ that are not real valued. Assuming this result, we are able to obtain bounds on the degree of these two characters; i.e., we will now prove Proposition 1.6.

Proposition 1.6. Let $\theta \in \operatorname{Irr}(U(13))$ be a character that is not real valued. Then we have $\theta(1) \leq 2^{16}$.

Proof. We write the equation of Proposition 3.1 for $U(13)$ :

$$
t(U(13))=\sum_{\nu_{2}=+1} \chi(1)-\sum_{\nu_{2}=-1} \chi(1)+0 \cdot \theta(1)+0 \cdot \bar{\theta}(1) .
$$

Add $2 \cdot \theta(1)$ to both sides and change the $\nu_{2}=-1$ term to be positive to obtain the inequality

$$
t(U(13))+2 \cdot \theta(1) \leq \sum_{\chi \in \operatorname{Irr}(U(13))} \chi(1) .
$$

This can be rearranged to yield

$$
\theta(1) \leq \frac{1}{2}\left\{\left[\sum_{\chi \in \operatorname{Irr}(U(13))} \chi(1)\right]-t(U(13))\right\}=2^{16},
$$

using Table 2

Remark 3.3. The bound obtained in Proposition [1.6 should be considered in light of the character degrees of $U(13)$ obtained by applying Slattery's algorithm: $U(13)$ has irreducible characters of degrees $\left\{1,2, \ldots, 2^{36}\right\}$. It is also possible to produce weak lower bounds (e.g., $\theta(1) \geq 4$ ) by using the "method of little groups" [3] to exhibit explicitly the characters of small degree and thereby show that they are real valued. Since we have not determined $\theta(1)$, we have elected not to give such computations.

\section{REMARKS}

The work in this paper was undertaken in the hope that it would lead to a proof that (for $k=\mathbb{F}_{2}$ ) the representations of $U(n)$ are all realizable over $\mathbb{R}$ for every $n$. As we have noted many times by now, there is no such proof: not all the representations of $U(n)$ are realizable over $\mathbb{R}$.

The results of this paper, however, suggest the further question (Problem 1.3) of whether every real character of $U(n)$ is afforded by a real representation. If every real character is so afforded, then we would obtain as a corollary the following stronger version of Proposition 1.6. 
Conjecture 4.1. If $\theta \in \operatorname{Irr}(U(13))$ is a character that is not real valued, then $\theta(1)=2^{16}$.

Conversely, this conjecture implies that every real character of $U(13)$ is afforded by a real representation.

Since the conjecture regards a single character of a finite group, it is perhaps surprising that we cannot perform a quick computation to resolve it. Readers familiar with the Atlas 2] of finite simple groups will be aware that the character tables of some very large finite simple groups have been computed.

However, finite simple groups typicially have few conjugacy classes (relative to other groups of the same order), and hence small character tables. The character tables of $p$-groups, however, tend to be very large. In addition, the strong structural restrictions on finite simple groups that make it possible to determine conjugacy classes and character tables do not exist for $p$-groups.

While there are general algorithms to compute character tables of finite groups, the time required to compute the character table of $U(n)$ grows very rapidly as a function of $n$. For example, the ratio of the time required for the character table of $U(6)$ to the time required for the character table of $U(5)$ has order of magnitude $10^{4}$. A direct computation of the entire character table of $U(13)$ is therefore not feasible. Thus it seems that whatever computation resolves the conjecture above will have to be specifically adapted to the group and character in question.

\section{REFERENCES}

1. Bosma, W., Cannon, J., and Playoust, C. The Magma algebra system. I. The user language. Computational algebra and number theory (London, 1993). J. Symbolic Comput. 24 (1997), no. 3-4, pp. 235-265. MR.98f:68006

2. Conway, J. H., Curtis, R. T., Norton, S. P., Parker, R. A., Wilson, R. A. Atlas of finite groups. (with computational assistance from J. G. Thackray.) Oxford University Press, Eynsham, 1985. MR88g:20025

3. Curtis, C. and Reiner, I. Methods of representation theory. Vol. I. With applications to finite groups and orders. Reprint of the 1981 original. John Wiley \& Sons, Inc., New York, 1990. MR.90k:20001

4. Isaacs, I. M., Character Theory of Finite Groups, Dover (1994).

5. Isaacs, I. M. and Karagueuzian, D. Conjugacy in groups of upper triangular matrices. J. Algebra 202 (1998), no. 2, pp. 704-711. MR99b:20011

6. Isaacs, I. M. and Karagueuzian, D. Erratum: "Conjugacy in groups of upper triangular matrices.” J. Algebra 208 (1998), no. 2, p. 722. MR99g:20021

7. Slattery, M. Computing character degrees in p-groups. J. Symbolic Comput. 2 (1986), no. 1, pp. 51-58. MR87e:20019

8. Vera López, A. and Arregi, J., Conjugacy classes in Sylow p-subgroups of GL $(n, q)$. J. Algebra 152 (1992), no. 1, pp. 1-19. MR 94b:20048

9. Vera López, A. and Arregi, J., private communication.

Mathematics Department, University of Wisconsin at Madison, Madison, Wisconsin 53706

E-mail address: isaacs@math.wisc.edu

Mathematics Department, Binghamton University, Binghamton, New York, 139026000

E-mail address: dikran@math.binghamton.edu 